\title{
Grampian Health Board's joint drug formulary
}

\author{
G Garvey, B Jappy, D Stewart, A Williams, P R S Duffus, J M Maitland, M J Valentine, \\ $\mathrm{S}$ Wedderburn, J Webster, J C Petrie
}

Department of Pharmacy, Grampian Health Board, Aberdeen

G Garvey, MB, pharmacist facilitator

B Jappy, Msc, principal pharmacist

D Stewart, MSC, pharmacist A Williams, MRPHARMS, chief administrative pharmaceutical officer

Clinical Pharmacology Unit, University of Aberdeen, Aberdeen AB2 4DQ

P R S Duffus, MRCGP, general practitioner

J M Maitland, MRCGP, general practitioner

M J Valentine, MRCGP, general practitioner $\mathrm{S}$ Wedderburn, MRCGP, general practitioner

J Webster, FRCP, senior lecturer

J C Petrie, FRCP, professor

Correspondence to:

Professor Petrie.

$\operatorname{BrMed} \mathcal{f} 1990 ; 301: 851-2$
Abstract

Objective-To develop a model for creating a joint general practice-hospital formulary, using the example of ulcer healing drugs.

Design-A joint formulary development group produced draft guidelines based on an earlier hospital formulary, which were sent to interested local general practitioners for consultation. Revised guidelines were then drawn up and forwarded to the health board's medicines committee for approval and distribution.

Setting-Grampian Health Board.

Subjects-Nine members of joint formulary development group plus local general practitioners who were invited to comment on a list of 11 ulcer healing drugs.

Main outcome measure-Degree of coincidence of drugs selected by hospital doctors and general practitioners.

Results - The ulcer healing drugs selected by the panel of general practitioners and by hospital doctors were highly coincident. The cost of one day's treatment with drugs varied considerably between hospital and general practice-for example, one drug cost 46p in hospital and $£ 1$ in general practice and another cost $£ 1.26$ in hospital and $£ 1.01$ in general practice. Overall, six drugs cost more in hospital and five cost more in general practice.

Conclusions - A joint formulary for use in hospitals and general practice in a health board can be devised fairly simply by consultation as virtually the same drugs are used in both types of practice. It should influence the health board's expenditure on drugs and affect the choice of drugs when a patient is discharged from hospital or is referred to any hospital in the region.

\section{Introduction}

Expenditure on drugs constitutes a large proportion of the cost of providing family practitioner services. ${ }^{1}$ The choice of drugs is usually assessed in terms of efficacy, safety, and cost. ${ }^{2}$ One of the aims outlined in the white paper Working for Patients is to ensure that the costs of prescribed drugs are kept within reasonable limits. ${ }^{3}$ The working paper Indicative Prescribing Budgets for General Practitioners states that it will be a responsibility of regional health authorities to encourage the development of joint formularies by family practitioner committees and district health authorities. ${ }^{4}$ Local formularies should help hospital doctors and general practitioners to use the same medicines, thus facilitating continuity of treatment between hospitals and the community. ${ }^{5}$

In Grampian Health Board a drug formulary for hospitals and information notes were developed together with a computer assisted feedback system for prescribers to audit compliance with agreed prescribing guidelines in each ward ${ }^{67}$ In addition, a pilot study of repeat prescribing in selected local general practices showed a considerable coincidence with the recommendations of the drug formulary for hospitals. There was sufficient common ground and good will to encourage an investigation of the feasibility of producing a joint Grampian Health Board drug formulary. ${ }^{8}$ We report a method that we developed to revise and develop a joint drug formulary, which covers recommendations by hospitals and general practitioners for prescribing each group of drugs.

\section{Methods}

The joint formulary development group was set up by the medicines committee of Grampian Health Board. Its members comprised four local general practitioners nominated by the area general practice subcommittee, three pharmacists including a pharmacist facilitator, and two clinical pharmacologists. The general practitioners in the development group with the assistance of the local faculty of the Royal College of General Practitioners wrote to 50 randomly selected principals in general practice in Grampian to ask them whether they wanted to receive the existing formulary recommendations for hospitals. After this survey local general practitioners were invited to comment on draft guidelines for each group of drugs to be included in the joint formulary.

The preparation of the draft text and graphic information for the joint formulary was coordinated by Aberdeen Royal Infirmary's pharmacy department after extensive consultation with specialists and high users in hospitals for each therapeutic category or group of drug. ${ }^{67}$ The list of suggested drugs for use in hospital was discussed by the joint formulary group and then sent for consultation (and for drugs to be added or deleted) to members of the volunteer panel of general practitioners who had declared an interest in the therapeutic category. They were asked for a considered reply within four weeks after discussion within their practices. Information was provided to these general practitioners on the comparative costs in general practice of drugs in the therapeutic group; the most commonly prescribed drugs in the drug group in Scotland; and any other relevant background information.

The replies from these general practitioners were then collated by the pharmacist facilitator. A revised version of the joint formulary recommendations for general practice and hospitals was prepared and discussed by the joint formulary group and forwarded to the medicines committee for approval and distribution. During these consultation stages we looked for a degree of coincidence in prescribing recommendations for hospital and general practice, taking into account the opinions of the practices and hospitals on efficacy, safety, usage, and relative costs. 


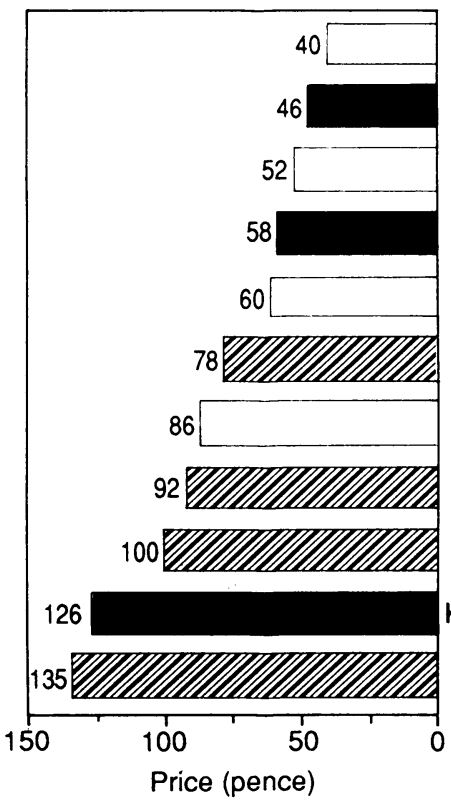

Drug

Treatment in general practice
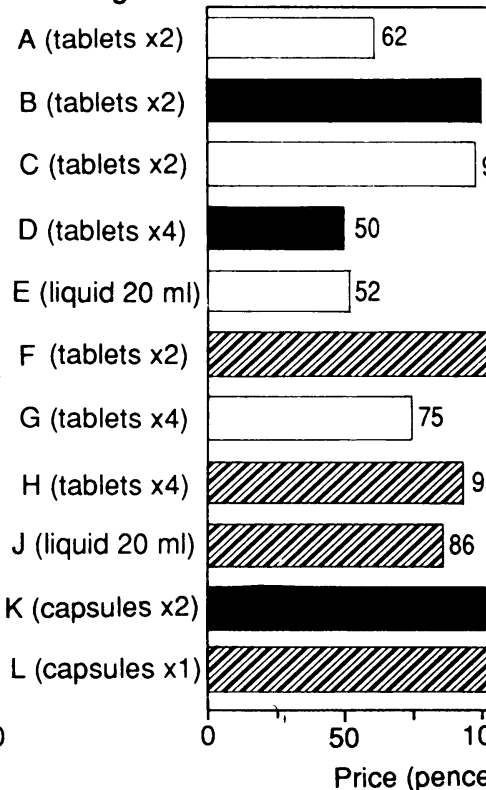

00

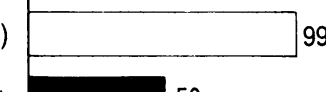

99
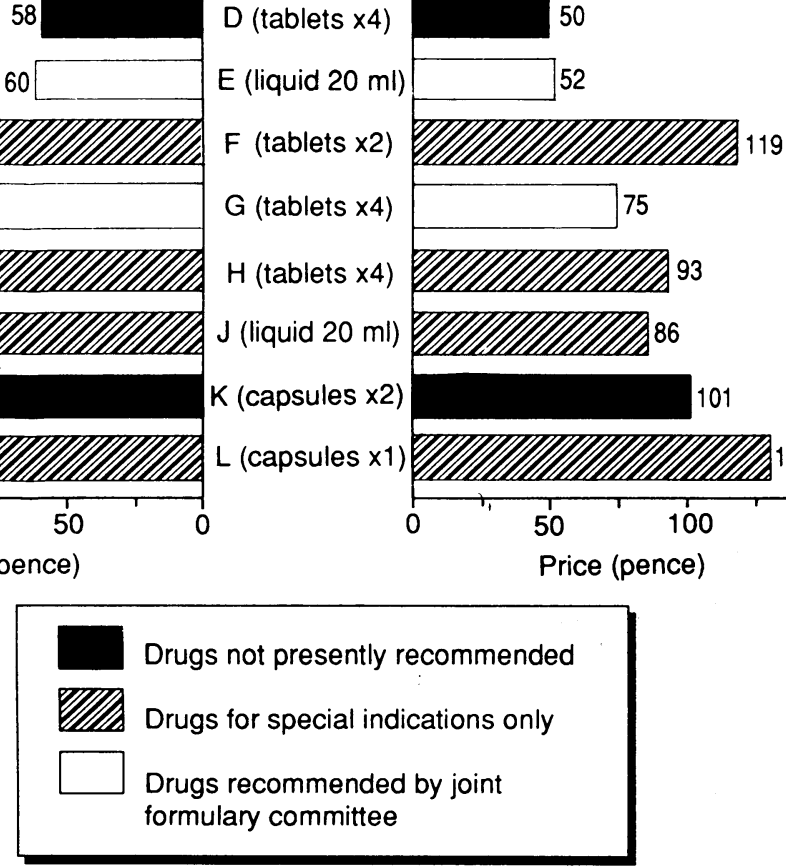

Cost of one day's treatment with ulcer healing drugs in hospital and general practice. Drugs were recommended by hospital and general practice subgroups of medicines committee after consultation with specialists and general practitioners

\section{Results}

In the pilot study 48 of the 50 randomly selected general practitioners wanted to receive the original drug formulary recommendations for hospitals. A total of 60 general practitioners volunteered to help produce the joint drug formulary.

The first therapeutic category that we chose to revise comprised drugs recommended for treatment of the gastrointestinal system. Nine information sheets were prepared after consultation. The figure shows, using the example of ulcer healing drugs, the cost of one day's treatment with drugs selected by both the hospitals and general practitioners. The doctors in Grampian are provided with the cost of the drugs but the drug names are not shown in the figure because the costs vary according to local or regional contracts and over time. The coincidence of the drugs selected by the panel of general practitioners and by hospital doctors is evident. It was also seen across the other gastrointestinal therapeutic categories and drug groups (antacids, antidiarrhoeal drugs, drugs for inflammatory bowel disease). For the four categories the revisions for the joint formulary recommendations entailed six additions or deletions to the previous hospital formulary. ${ }^{6}$ Important differences in cost of the four groups between hospital and general practice was evident: a preparation sometimes cost almost four times more in general practice, though sometimes it cost less.

\section{Discussion}

Drug formularies have met with mixed results across the health service. ${ }^{2}$ The white paper Working for Patients $^{3}$ and associated working papers together with other initiatives such as the funding of national medicines resource centres and the guidelines of the Royal College of General Practitioners on preparing practice formularies ${ }^{9}$ have provided an incentive and resource to develop rational guidelines for prescribing both in hospitals and in individual practices.

We took the development of a formulary one step further. The voluntary collaborative production by hospitals and general practitioners of guidelines, by therapeutic category, for prescribing drugs in a health board could have a major impact on the pattern and cost of prescribing. Doctors in hospitals, all principals in general practice, and senior medical students now receive jointly prepared authoritative information supplied to them by Grampian Health Board; the principal categories covered in the earlier hospital formulary ${ }^{6}$ should be completed by early next year.

We found that general practitioners welcomed these local prescribing guidelines as providing authoritative statements about the selection of drugs based on considerations including efficacy, safety, usage, and cost. We also found that general practitioners wanted to help revise the guidelines, according to their particular knowledge. The revised guidelines have already highlighted several issues such as the similarity of choice of drugs by hospital doctors and general practitioners when there is a freedom to choose. The model system, as with other guidelines such as the World Health Organisation's essential drugs lists, offers the flexibility for different practices to accept a variable "core" of the joint formulary and to exercise such choice as they want to make on individual selections. Thus they can adapt this core to give a formulary for their own requirements.

One factor in the selection of drugs (but not the major one) is the cost of an equivalent daily dose of comparable drugs. ${ }^{2}$ In a recent survey only a third of general practitioners estimated the correct price of drugs (to within $25 \%$ ), and they requested information on drug prices. ${ }^{10}$ Our joint formulary guidelines should increase prescribers' awareness of costs of drugs and the relative and often strikingly different costs between general practice and hospitals, where contracts, discounts, and loss leaders may be factors. This information should influence the health board's overall expenditure on drugs, which is the lowest in Scotland after that of the Highland and the Orkney and Shetland Health Boards. ${ }^{4}$ It should also affect the choice of drugs when a patient is discharged from hospital or is referred to any hospital in Grampian.

Audit through computer assisted monitoring of compliance with self selected prescribing guidelines is well established in Grampian hospitals. ${ }^{7}$ The availability of Scottish prescribing analysis (SPA) data, which are similar to the prescribing analysis and cost (PACT data in England, for general practitioners facilitates audit of prescribing and its coincidence with local prescribing guidelines. Such feedback will be used by the joint formulary group in future revisions of the formulary. The value of the pharmacist facilitator in implementing such an approach has been apparent throughout this project.

We thank the Scottish Home and Health Department for supporting GG. DS was a Medical Research Council advanced course student in clinical pharmacology.

1 O'Brien B. Indicative drug budgets for general practitioners. $\mathrm{Br}$ Med $\mathcal{J}$ 1989;298:944-6.

2. Petrie JC, Scott AK. Drug formularies. Br Med f 1987;294:919-20.

3 Secretaries of State for Health, Wales, Northern Ireland, and Scotland. Working for patients. London: HMSO, 1989.

4 Scottish Home and Health Department. Indicative prescribing budgets. Edinburgh: SHHD, 1990. (Working paper 4.)

5 Scottish Home and Health Department. Implications for primary health care services. London: HMSO, 1989. (Scottish working paper 1 )

6 Jappy B, Krska J, Downie G, Smith ME, Williams A, Petrie JC. The Grampian hospitals formulary. Health Bulletin 1989;47:223-6.

7 Krska J, Williams A, Jappy B, Petrie JC. Use of a computerised stock control system to introduce and monitor a hospital formulary. Pharmaceutical fournal 1986;237:559.

8 Stewart D. A comparative study of repeat prescribing in general practice with hospital recommendations. Aberdeen: University of Aberdeen, 1989 $122 \mathrm{pp}$. (MSc thesis.)

9 Waine C. How to produce a practice formulary. London: Royal College of General Practitioners, 1989.

10 Ryan M, Yule B, Bond C, Taylor RJ. Scottish general practitioners' attitudes and knowledge in respect of prescribing costs. Br Med $\mathcal{f}$ 1990;300:1316-8.

(Accepted 1 August 1990) 